\title{
Aseptic Meningitis in Hereditary Autoinflammatory Diseases
}

\author{
Antonis Neokleous ${ }^{1}$, Savvas Psarelis ${ }^{2}$, Konstantinos M. Parperis ${ }^{1,3}$ \\ 1. Internal Medicine, University of Cyprus Medical School, Nicosia, CYP 2. Division of Rheumatology, Nicosia General \\ Hospital, Nicosia, CYP 3. Division of Rheumatology, University of Arizona College of Medicine, Phoenix, USA
}

Corresponding author: Antonis Neokleous, aneokl01@ucy.ac.cy

\begin{abstract}
Autoinflammatory diseases (ADs) refer to a group of disorders of the innate immune system, mainly monogenic, marked by episodes of systemic inflammation. Aseptic meningitis is a rare neurological manifestation of ADs characterized by meningeal inflammation, negative routine cultures in the cerebrospinal fluid and identical signs and symptoms of bacterial meningitis. Herein, the aim of this review article is to describe the association between aseptic meningitis and ADs, especially in patients with familial Mediterranean fever (FMF) and chronic infantile neurological cutaneous articular (CINCA) syndrome. We will discuss the emerging role of proinflammatory cytokines, such as interleukin 1 (IL-1), interleukin 6 (IL-6) and tumor necrosis factor-alpha (TNF- $\alpha$ ), in the pathogenesis of aseptic meningitis in ADs, and will explore recent treatment developments, such as the use of biological agents.
\end{abstract}

Received 04/17/2019

Review began 04/22/2019 Review ended 05/14/2020 Published 05/22/2020

๑ Copyright 2020

Neokleous et al. This is an open access article distributed under the terms of the Creative Commons Attribution License CC-BY 4.0., which permits unrestricted use, distribution, and reproduction in any medium, provided the original author and source are credited.
Categories: Rheumatology

Keywords: autoinflammatory diseases, familial mediterranean fever, chronic infantile neurological cutaneous articular syndrome, aseptic meningitis, interleukin 1, interleukin 6, cryopyrin-associated periodic syndrome, neurological manifestations

\section{Introduction And Background}

Autoinflammatory diseases (ADs) are systemic disorders of the innate immune system, characterized by repeated episodes of inflammation, without the presence of autoantibodies or reactive $\mathrm{T}$ cells. An overactive innate immune system plays a key role in the pathogenesis of these disorders through the increased production and activity of interleukin $1 \beta$ (IL-1 $\beta$ ) and interleukin 6 (IL-6) [1]. So far, there are 12 known monogenic ADs: (1) familial Mediterranean fever (FMF), (2) tumor necrosis factor receptor-associated periodic (TRAPS) syndrome and the cryopyrin-associated periodic syndromes (CAPS) that comprises the (3) familial cold autoinflammatory syndrome (FCAS), (4) Muckle-Wells syndrome (MWS) and (5) chronic infantile neurological cutaneous articular (CINCA) syndrome. Other ADs include the (6) mevalonate kinase deficiency (MKD), (7) NLRP12-associated autoinflammatory disorder, (8) Blau syndrome (BS), (9) early-onset sarcoidosis, (10) pyogenic arthritis-pyoderma gangrenosum and acne (PAPA) syndrome, (11) Majeed syndrome (MS) and (12) deficiency of the interleukin 1 (IL-1) receptor antagonist. Each of these conditions may manifest itself with a wide array and severity of systemic and organ-specific symptoms and usually they have been associated with elevated inflammatory biomarkers [2]. ADs are generally marked by an early onset in the first year of life or early childhood; however, adult onset has also been described, particularly in FMF and TRAPS [3-5]. Many of these syndromes are hereditary and result from a single gene mutation (Table 1) [5]. 


\section{Cureus}

\begin{tabular}{|c|c|c|c|}
\hline & Gene & Mutated Protein & Inheritance \\
\hline \multicolumn{4}{|l|}{ Monogenic periodic fevers } \\
\hline FMF & MEFV & Pyrin/marenostrin & AR \\
\hline MKD & MVK & Mevalonate kinase & $A R$ \\
\hline TRAPS & TNFRSF1A & TNFRSF1A & $A D$ \\
\hline \multicolumn{4}{|l|}{ Cryoporin-associated periodic syndromes } \\
\hline CINCA & & & AD, sporadic \\
\hline FCAS & NRLP3/CIAS1 & Cryopyrin & $A D$ \\
\hline MWS & & & $A D$ \\
\hline NLRP12-associated autoinflammatory disorder & NLRP12 & NLRP12 & $A D$ \\
\hline \multicolumn{4}{|l|}{ Autoinflammatory granulomatous disorders } \\
\hline BS & NOD2/CARD15 & NOD 2(CARD15) & $A D$ \\
\hline Early-onset sarcoidosis & NOD2/CARD15 & NOD 2(CARD15) & Sporadic \\
\hline \multicolumn{4}{|l|}{ Autoinflammatory pyogenic disorders } \\
\hline DIRA & IL1RN & Interleukin 1 receptor antagonist & AR \\
\hline MS & LPIN2 & Lipin-2 & AR, sporadic \\
\hline PAPA & PSTPIP1 (CD2BP1) & PSTPIP1 (CD2BP1) & $A D$ \\
\hline
\end{tabular}

\section{TABLE 1: Classification of the Monogenic Autoinflammatory Syndromes}

FMF: familial Mediterranean fever, MKD: mevalonate kinase deficiency, TRAPS: tumor necrosis factor receptor-associated periodic syndrome, CINCA: chronic infantile neurological cutaneous articular syndrome, FCAS: familial cold autoinflammatory syndrome, MWS: Muckle-Wells syndrome, BS: Blau syndrome, DIRA: deficiency of interleukin 1 receptor antagonist, MS: Majeed syndrome, PAPA: pyogenic arthritis pyoderma gangrenosum and cystic acne syndrome, NLRP: NACHT domain-, leucine-rich repeat- and pyrin domain-containing protein, AD: autosomal dominant, AR: autosomal recessive [5]

To achieve an accurate diagnosis, a number of steps need to be taken, including detailed history taking, physical exam, laboratory studies and genetic testing. Characteristic clinical features of these disorders include periodic fevers, neutrophilic rashes or urticaria, polyserositis, polyarthralgia or polyarthritis, hepatosplenomegaly, lymphadenopathy, elevated acute phase reactants, neutrophilia and a long-term risk of secondary amyloidosis [6]. Moreover, these diseases may also present with neurological symptoms due to aseptic meningitis $[7,8]$. The most frequent conditions associated with aseptic meningitis appear to be the CIGNA syndrome, an $\mathrm{AD}$ that belongs to the family of the CAPS. In particular, meningitis can be the initial manifestation in FMF and CINCA syndrome; however, it can occur at any stage of the disease. A potential pathophysiologic mechanism highlights the role of IL-1, IL-6 and tumor necrosis factor-alpha (TNF- $\alpha$ ) in the pathogenesis of aseptic meningitis in FMF and CINCA syndrome [9-11].

\section{Review}

\section{The pathophysiology of ADs}

The immune system contains a complex armamentarium of cells and signaling molecules, which can combat infection, eradicate foreign agents and promote repair. Over time, the immune system, which comprises the innate and adaptive immunity, has evolved to provide effective protection against infectious diseases [12]. The innate immune system is characterized by nonspecific defense mechanisms against foreign antigens soon after their appearance, while the adaptive immunity comprises specific recognition of pathogens before a response is initiated. IL-1, a signaling molecule of the innate immune system and a proinflammatory cytokine, is typically overexpressed following injury or infection, and through its receptor, it may affect a wide variety of cell types within the body [13]. IL-1 belongs to a family of cytokines comprising 11 different isoforms, though most is known about IL- $1 \alpha$ and $1 \beta$ because of their central role in regulation of inflammation. IL- $1 \beta$ is expressed as a precursor protein that is then cleaved by caspase- 1 , a protein that forms a part of a complex known as the inflammasome, producing the active IL-1 $\beta$. When the balance of the immune response is distorted, it can promote inflammation and the pathogenesis of various diseases. Overproduction of IL- $1 \beta$ is associated with a variety of autoinflammatory syndromes, specifically FMF and 
CINCA syndrome. Some are associated with mutations within NACHT domain-, leucine-rich repeat- and pyrin domain-containing protein 3 (NLRP3), which is a gene that encodes cryopyrin, a component of the inflammasome. These mutations are thought to increase the expression of the active interleukin $1 \beta$ promoting abnormal activity of this signaling molecule that may result in the development of FMF and CINCA syndrome [14].

\section{Aseptic meningitis as a neurological complication in ADs}

Previous studies demonstrated that the production of proinflammatory cytokines such as TNF- $\alpha$, IL- 6 and IL- $1 \beta$ by monocytes of the central nervous system (CNS) seems to play an important role in the alteration of the blood-cerebrospinal fluid (CSF) barrier [15,16]. Although the mechanism of action is still not fully defined, experimental studies in meningitis induced by infection suggest that these cytokines are directly cytotoxic to the neurons. Furthermore, the cytotoxicity can occur indirectly, through the release of oxygen free radicals, which attack the double bonds of polyunsaturated fatty acids on cellular membranes in the process of lipid peroxidation of the membrane [17].

The proinflammatory cytokines, together or independently, are being responsible for the first steps of the inflammatory cascade that results in organ damage. Patients with ADs who develop aseptic meningitis present with classic symptoms of meningeal inflammation, including nausea, headache, fever and neck stiffness. The clinical course of aseptic meningitis varies from acute, self-limited episode to chronic or recurrent disease, and when meningitis is the initial manifestation of the $\mathrm{AD}$ it might pose a diagnostic challenge [18]. The diagnosis is based on the characteristic signs and symptoms of meningitis, after ruling out alternative diseases. It is critical for health care providers to exclude other etiologies such as a bacterial or fungal infection or the use medications that can induce aseptic meningitis, for example nonsteroid antiinflammatory drugs [19]. The diagnostic evaluation includes lumbar puncture with CSF analysis, electroencephalogram, evoked-potential studies, CT and MRI of the brain. The results of these studies will allow the clinician to thoroughly evaluate the patient with symptoms of meningitis [20]. For the patients who experienced persistent symptoms of aseptic meningitis associated with fever, and negative infectious diseases workup, we would recommend genetic testing for ADs. In most patients with aseptic meningitis and autoinflammatory syndrome the CSF analysis may yield pleocytosis with elevated protein and normal glucose levels [18].

\section{Aseptic meningitis in FMF}

FMF is a monogenic autosomal recessive disease marked by recurrent episodes of fever, polyserositis, arthritis and erysipelas-like erythema [21]. It is caused by mutations in the MEFV gene that encodes the protein pyrin $[22,23]$. This protein is made up of 781 amino acids and is expressed mostly in neutrophils, eosinophil granulocytes, monocytes, fibroblasts of the skin, peritoneum and synovial. Pyrin mutations cause changes in inflammasome function, which lead to increased synthesis of proinflammatory cytokines, (mainly IL-1 $\beta$ and IL-6) and altered inhibition of apoptosis. MEFV mutation might contribute to the inflammatory phenotype in the presence of other genetic or environmental factors, through IL-6 hypersecretion [24]. Microglia, astroglia or both are the possible sources of IL- 6 in the CNS. The CNS hypersecretion of IL-6 may cause increased blood brain-barrier permeability, leading to subtle edema, CSF inflammation with increased intracranial pressure and inner ear damage [25].

From the clinical point perspective, different FMF genotype and phenotypes have been identified [26]. The typical FMF phenotype (mutations in MEFV exon 10) is characterized by recurrent inflammatory episodes. During these episodes, patients mainly complain of fever and abdominal pain. Patients with the incomplete FMF phenotype (mutations in MEFV exons 2 or 3) present with uncommon manifestations such as fever and headache due to aseptic meningitis [8,27]. Meningitis, in FMF, follows a similar pattern with the fever, with self-limited episodes, lasting three to five days [23]. In terms of treatment, colchicine is considered as the gold standard for patients with FMF. Other treatment strategies, in patients who fail to respond to colchicine, include the use of biologics, specifically the IL- 1 inhibitors and TNF-a blockers. Examples include the anakinra, an IL-1 receptor inhibitor, the canakinumab, an anti-IL-1 $\beta$ monoclonal antibody, the rilonacept, an IL-1 receptor fusion protein, the infliximab, a TNF-a monoclonal antibody and the etanercept, a TNF dimeric fusion protein [28].

\section{Aseptic meningitis in CAPS and CINCA syndrome}

CAPS are a group of ADs transmitted by autosomal dominant inheritance caused by mutations in the NLRP3 gene, encoding cryopyrin, an inflammasome protein that directly activates IL-1 $\beta$. This mutation induces an overexpression of IL-1 $\beta$, leading to episodic fever associated with organ-specific inflammatory symptoms. There are three known forms of CAPS: the familial cold auto-inflammatory syndrome (FCAS), the MuckleWells syndrome (MWS) and the CINCA syndrome, which is the most severe form (Table 2) [24,29-31]. 


\section{Cureus}

\begin{tabular}{|l|l|l|}
\hline FCAS & MWS & CINCA \\
\hline Inheritance & AD & AD, sporadic \\
\hline AD & Main clinical features & \\
Cold-induced: & Fever & Fever \\
-Fever & Urticarial rash & Urticarial rash \\
-Rash & Conjuctivitis & Conjuctivitis \\
-Conjunctivitis & Arthralgia & Visual and intellectual damage \\
-Arthralgia & Sensorineural deafness & Sensorineural deafness \\
& AA amyloidosis (in 25\% of patients) leading to renal failure & Progressive chronic meningitis \\
& & Destructive arthritis \\
& & AA amyloidosis leading to renal failure \\
\hline
\end{tabular}

\section{TABLE 2: Cryopyrin-Associated Periodic Syndrome (CAPS)}

FCAS: familial cold autoinflammatory syndrome, MWS: Muckle-Wells syndrome, CINCA: chronic infantile neurological cutaneous articular syndrome, AD: autosomal dominant, AA: amyloid A [24]

In a cohort study by Parker et al., the investigators showed that three out of four patients with CAPS with available CSF data had evidence of aseptic meningitis [32]. Although meningitis can occur in all three types of CAPS, meningitis in CINCA syndrome patients is more common. CINCA syndrome may manifest with fever, urticaria, sensorineural hearing loss, uveitis, lymphadenopathy, hepatosplenomegaly papilledema, optic nerve atrophy leading to blindness, mental retardation, deforming osteoarthropathy of the large joints, hypertrophy of growth plates and chronic meningitis [32]. Chronic aseptic meningitis, defined as at least one month of unresolved inflammation of the CSF, might result in increased intracranial pressure,

ventriculomegaly, cerebral and optic nerve atrophy. Given that elevated levels of IL-1 play an important role in the disease pathogenesis of CAPS, clinician can consider the use IL-1 inhibitors in order to control inflammation. Anakinra was the first drug utilized in these patients, resulting in significant improvement in neurological symptoms [33-35]. Furthermore, a follow-up study showed that in patients treated with anakinra, the CSF levels of cytokines IL-1 and IL-6 and monocyte and granulocyte counts were reduced significantly [31]. Alternatively, in patients who failed or did not tolerated anakinra, canakinumab and rilonacept monoclonal antibodies against IL-1 $\beta$, might be alternative therapeutic options [11,36-38]. To our knowledge, there are no approved biologic disease-modifying antirheumatic drugs for aseptic meningitis in ADs.

\section{Conclusions}

The pathogenetic mechanisms of aseptic meningitis in ADs remain unclear; thus, no consensus exists regarding the optimal treatment and prognosis. Future studies are required to elucidate pathophysiology of aseptic meningitis associated with ADs in order to develop treatment strategies, and improve patient outcomes and prognosis. Physicians should be aware of this manifestation to identify early the disease and treat appropriately. From a therapeutic point of view, no evidence-based therapy exists for aseptic meningitis in $\mathrm{ADs}$; however colchicine and corticosteroids are often used to treat symptoms in ADs, with varying results. Recent advances for the development of biological agents, such as anti-TNF-a, anti-IL-1 and anti-IL-6, have opened up new therapeutic options for the management of these heterogeneous disorders.

\section{Additional Information}

\section{Disclosures}

Conflicts of interest: In compliance with the ICMJE uniform disclosure form, all authors declare the following: Payment/services info: All authors have declared that no financial support was received from any organization for the submitted work. Financial relationships: All authors have declared that they have no financial relationships at present or within the previous three years with any organizations that might have an interest in the submitted work. Other relationships: All authors have declared that there are no other relationships or activities that could appear to have influenced the submitted work. 


\section{References}

1. Montealegre Sanchez GA, Hashkes PJ: Neurological manifestations of the Mendelian-inherited autoinflammatory syndromes. Dev Med Child Neurol. 2009, 51:420-428. 10.1111/j.1469-8749.2009.03336.x

2. Caso F, Rigante D, Vitale A, et al.: Monogenic autoinflammatory syndromes: state of the art on genetic, clinical, and therapeutic issues. Int J Rheumatol. 2013, 2013:513782. 10.1155/2013/513782

3. Lamkanfi M, Dixit VM: Inflammasomes and their roles in health and disease. Annu Rev Cell Dev Biol. 2012, 28:137-161. 10.1146/annurev-cellbio-101011-155745

4. Cantarini L, Capecchi PL, Lucherini OM, Laghi Pasini F, Galeazzi M: Familial Mediterranean fever diagnosed in an elderly patient. Clin Exp Rheumatol. 2010, 28:91.

5. Sayarlioglu M, Cefle A, Inanc M, et al.: Characteristics of patients with adult-onset familial Mediterranean fever in Turkey: analysis of 401 cases. Int J Clin Pract. 2005, 59:202-205. 10.1111/j.1742-1241.2004.00294.x

6. Cush JJ: Autoinflammatory syndromes. Dermatol Clin. 2013, 31:471-480. 10.1016/j.det.2013.05.001

7. Salsano E, Rizzo A, Bedini G, et al.: An autoinflammatory neurological disease due to interleukin 6 hypersecretion. J Neuroinflamm. 2013, 10:29. 10.1186/1742-2094-10-29

8. Nikiphorou E, Neocleous V, Phylactou LA, Psarelis S: Successful use of tocilizumab in two cases of severe autoinflammatory disease with a single copy of the Mediterranean fever gene. Rheumatology. 2017, 56:1627-1628. 10.1093/rheumatology/kex180

9. Krebs VLJ, Okay TS, Okay Y, Vaz FAC: Tumor necrosis factor- $\alpha$, interleukin- $1 \beta$ and interleukin- 6 in the cerebrospinal fluid of newborn with meningitis. Arq Neuropsiquiatr. 2005, 63:7-13. 10.1590/s0004$282 \times 2005000100002$

10. Ozdogan H, Ugurlu S: Familial Mediterranean fever. La Presse Med. 2019, 48:e61-e76. 10.1016/j.lpm.2018.08.014

11. Keddie S, Parker T, Lachmann HJ, Ginsberg L: Cryopyrin-associated periodic fever syndrome and the nervous system. Curr Treat Options Neurol. 2018, 20:43. 10.1007/s11940-018-0526-1

12. Hato T, Dagher PC: How the innate system senses trouble and causes trouble . Clin J Am Soc Nephrol. 2015, 10:1459-1469. 10.2215/CJN.04680514

13. Dinarello CA: Overview of IL-1 family in innate inflammation and acquired immunity . Immunol Rev. 2018, 281:8-27. 10.1111/imr.12621

14. Masters SL, Simon A, Aksentijevich I, Kastner DL: Horror autoinflammaticus: the molecular pathophysiology of autoinflammatory disease. Annu Rev Immunol. 2009, 27:621-668. 10.1146/annurev.immunol.25.022106.141627

15. Quagliarello VJ, Wispelwey B, Long WJ Jr, Scheld WM: Recombinant human interleukin-1 induces meningitis and blood-brain barrier injury in the rat, characterization and comparison with tumor necrosis factor. J Clin Invest. 1991, 87:1360-1366. 10.1172/JCI115140

16. Ramilo O, Sáez-Llorens X, Mertsola J, et al.: Tumor necrosis factor $\alpha /$ cachectin and interleukin $1 \beta$ initiate meningeal Inflammation. J.Exp Med. 1990, 172:497-507. 10.1084/jem.172.2.497

17. Glimäker M, Kragsbjerg P, Forsgren M, Olcén P: Tumor necrosis factor- $\alpha$ (TNF- $\alpha$ ) in cerebrospinal fluid from patients with meningitis of different etiologies: high levels of TNF- $\alpha$ indicate bacterial meningitis. J Infect Dis. 1993, 167:882-889. 10.1093/infdis/167.4.882

18. Ucelli A, Gattorno M: Neurological Manifestations in autoinflammatory diseases. Clin Exp Rheumatol. 2018, 36:61-67.

19. Nakamura M, Niino M: Chapter4: Meningitis Associated with Autoimmune Diseases. pp. 175-201 .

20. Appenzeller S, Kobayashi E, Costallat LT, et al.: Magnetic resorance imaging in the evaluation of patients with aseptic meningoencephalitis and connective tissue disorders. Arq Neuropsiquiatr. 2000, 58:45-51. 10.1590/S0004-282X2000000100007

21. Lidar M, Livneh A: Familial Mediterranean fever: clinical, molecular and management advancements . Neth J Med. 2007, 65:318-324.

22. Milhavet F, Cuisset L, Hoffman HM, et al.: The infevers autoinflammatory mutation online registry: update with new genes and functions. Hum Mutat. 2008, 29:803-808. 10.1002/humu.20720

23. French FMF Consortium: A candidate gene for familial Mediterranean fever . Nat Genet. 1997, 17:25-31. 10.1038/ng0997-25

24. Aksentijevich I, Kastner DL: Genetics of monogenic autoinflammatory diseases: past successes, future challenges. Nat Rev Rheumatol. 2011, 7:469-478. 10.1038/nrrheum.2011.94

25. Goldbach-Mansky R, Dailey NJ, Canna SW, et al.: Neonatal-onset multisystem inflammatory disease responsive to interleukin-1beta inhibition. N Engl J Med. 2006, 355:581-592. 10.1056/NEJMoa055137

26. Camus D, Shinar Y, Aamar S, et al.: Silent carriage of two familial Mediterranean fever gene mutations in large families with only a single identified patient. Clin Genet. 2012, 82:288-291. 10.1111/j.13990004.2011.01785.x

27. Karachaliou I, Karachalios G, Charalabopoulos A, Charalabopoulos K: Meningitis associated with familial Mediterranean fever. Int J Clin Pract Suppl. 2005, 147:60-61. 10.1111/j.1368-504x.2005.00290.x

28. Feld O, Yahalom G, Livneh A: Neurologic and other systemic manifestations in FMF: published and own experience. Best Pract Res Clin Rheumatol. 2012, 26:119-133. 10.1016/j.berh.2012.01.004

29. Cantarini L, Lucherini OM, Frediani B, et al.: Bridging the gap between the clinician and the patient with cryopyrin-associated periodic syndromes. Int J Immunopathol Pharmacol. 2011, 24:827-836. 10.1177/039463201102400402

30. Agostini L, Martinon F, Burns K, McDermott MF, Hawkins PN, Tschopp J: NALP3 forms an IL-1区-processing inflammasome with increased activity in Muckle-Wells autoinflammatory disorder. Immunity. 2004, 20:319325. 10.1016/s1074-7613(04)00046-9

31. Finetti M, Omenetti A, Federici S, Caorsi R, Gattorno M: Chronic infantile neurological cutaneous and articular (CINCA) syndrome: a review. Orphanet J Rare Dis. 2016, 11:167. 10.1186/s13023-016-0542-8

32. Parker T, Keddie S, Kidd D, et al.: Neurology of the cryopyrin-associated periodic fever syndrome . Eur J Neurol. 2016, 23:1145-1151. 10.1111/ene.12965

33. Novroski AR, Baldwin KJ: Chronic autoimmune meningoencephalitis and periodic fever syndrome treated 


\section{Cureus}

with anakinra. Case Rep Neurol. 2017, 9:91-97. 10.1159/000472147

34. Rigante D, Ansuini V, Caldarelli M, Bertoni B, Torraca IL, Stabile A: Hydrocephalus in CINCA syndrome treated with anakinra. Childs Nerv Syst. 2006, 22:334-337. 10.1007/s00381-006-1280-3

35. Rodriguez-Smith J, Lin YC, Tsai WL, et al.: CSF cytokines correlate with aseptic meningitis and blood brain barrier function in Neonatal Onset Multisystem Inflammatory Disease (NOMID). Arthritis Rheumatol. 2017, 69:1325-1336. 10.1002/art.40055

36. Hoffman HM, Throne ML, Amar NJ, et al.: Efficacy and safety of rilonacept (interleukin-1 Trap) in patients with cryopyrin-associated periodic syndromes: results from two sequential placebo-controlled studies. Arthritis Rheum. 2008, 58:2443-2452. 10.1002/art.23687

37. Rigante D, Torraca IL, Avallone L, Pugliese AL, Gaspari S, Stabile A: The pharmacologic basis of treatment with colchicine in children with familial Mediterranean fever. Eur Rev Med Pharmacol Sci. 2006, 10:173178 .

38. Ter Haar N, Lachmann H, Ozen S, et al.: Treatment of autoinflammatory diseases: results from the Eurofever Registry and a literature review. Ann Rheum Dis. 2013, 72:678-685. 10.1136/annrheumdis-2011201268 